\title{
Further Study on Semi-prequasi-invex Type Programming
}

\author{
Keke $\mathrm{Li}^{1}$, Zaiyun Peng ${ }^{2 *}$, Yawei Liu² and Jing Zeng ${ }^{3}$ \\ ${ }^{1}$ School of Mathematical Sciences, Chongqing Normal University, Chongqing, P.R. China \\ ${ }^{2}$ College of Mathematics and Statistics, Chongqing Jiaotong University, Chongqing, P.R. China \\ ${ }^{3}$ College of Mathematics and Statistics, Chongqing Technology and Business University, Chongqing, P.R. China \\ Email: pengzaiyun@126.com
}

\begin{abstract}
The purpose of this paper is to study semi-prequasi-invex type multiobjective optimization problem with inequality constraints and generalized nonlinear fractional programming. Two alternative theorems and an optimality necessary condition for multiobjective optimization problem are obtained. Moreover, a strong duality theorem and a saddle point theorem for generalized nonlinear fractional programming are derived. Our results improve and generalize the corresponding ones in the literature.
\end{abstract}

Keywords: Semi-prequasi-invex functions, multiobjective optimization problem, generalized nonlinear fractional programming.

\section{Introduction and Preliminaries}

It is well known that convexity and generalized convexity play an important role in nonlinear programming, convex analysis, and vector optimization theory etc.. Therefore, there are many literatures studying on this subject. The characterizations for the classical invexity was considered by Ben-Israel and Mond [1], Martin [2]. In recent years, the properties and applications of preinvexity have been investigated by many authors (see [2], [3], [4], [5], [6], [7], [8]). In [9], Yang introduced the prequasi-invex type functions and discussed prequasi-invex type multiobjective optimization problem as applications. Yang et al. [10], Luo and $\mathrm{Xu}$ [11] presented some properties of prequasi-invex functions and discussed some of its applications in optimization problem. Recently, Peng et al. [12] studied characterizations and criterions of vector-valued $D$-semiprequasi-invex mappings and obtained an important application in vector optimization problem. Some characterizations and applications of semi-prequasi-invexity can be found in Zhao [13], Zhao et al. [14] and Mishra [15]. Under four sets assumptions Xu [16] discussed four theorems of strong duality.

Motivated and inspired by the results in [9], [13], [16], in this paper we mainly study the applications of semi-prequasi-invex type multiobjective optimization problem with inequality constraints and generalized nonlinear fractional programming. Two alternative theorems and an optimality necessary condition for multiobjective optimization problem are given. Moreover, by employing the alternative theorem, a strong duality theorem and a saddle point theorem for generalized nonlinear fractional programming are discussed. Our results improve the corresponding ones in [9], [13], [16].

Now, we recall some concepts about semi-prequasi-invex functions.

Definition 1. (see [3],[9]) A set $E \subseteq R^{n}$ is called to be semi-connected if there exists a vector function $\eta: E \times E \times[0,1] \rightarrow E$, such that

$$
x, y \in E, \lambda \in[0,1] \Rightarrow y+\lambda \eta(x, y, \lambda) \in E .
$$

Definition 2. (see [9]) Let $E \subseteq R^{n}$ be a semi-connected set with respect to $\eta: E \times E \times[0,1] \rightarrow E$. We call that $f: E \rightarrow R^{n}$ is semi-prequasi-invex if, for all $x, y \in E, \lambda \in[0,1]$,

$$
f(y+\lambda \eta(x, y, \lambda)) \leq \max \{f(x), f(y)\} .
$$

Example 3. This example illustrates the existence of semi-connected set. Let $E=[-1,1]$, and

$$
\eta(x, y, \lambda)= \begin{cases}x-y-\lambda, & 0 \leq x \leq 1,0 \leq y \leq 1 \\ x-y+\lambda, & -1 \leq x<0,-1 \leq y<0 \\ -\frac{3}{4}-y+\lambda, & 0<x \leq 1,-1 \leq y<0 \\ \frac{2}{3}-y-\lambda, & -1 \leq x<0,0 \leq y \leq 1 \\ -1-y+\lambda, & x=0,-1 \leq y<0\end{cases}
$$


Clearly, $E$ is a semi-connected set with respect to $\eta$.

Next, we give two properties of semi-prequasi-invex functions.

Theorem 4. Let $E \subseteq R^{n}$ be a semi-connected set with respect to $\eta: E \times E \times[0,1] \rightarrow E$, assume $f(x): E \rightarrow R$ is a semi-prequasi-invex function on $E$. Then, $\lambda f(x)$ is also a semi-prequasi-invex function on $E$ with respect to the same $\eta(x, y, \theta)$, where $\lambda \geq 0$.

Proof. Since $f(x)$ is a semi-prequasi-invex function on $E$, then for any $\theta \in[0,1]$ and $x, y \in E$, we have

$$
f(y+\theta \eta(x, y, \theta)) \leq \max \{f(x), f(y)\} .
$$

Note that $\lambda \geq 0$, then we get

$$
\lambda f(y+\theta \eta(x, y, \theta)) \leq \lambda \max \{f(x), f(y)\}=\max \{\lambda f(x), \lambda f(y)\},
$$

that is, $\lambda f(x)$ is also a semi-prequasi-invex function.

Theorem 5. Let $E \subseteq R^{n}$ be a semi-connected set with respect to $\eta(x, y, \theta)$, assume $g_{j}(x): E \rightarrow R(j=$ $1, \cdots, m)$, are semi-prequasi-invex functions on $E$ with respect to the same $\eta(x, y, \theta)$. Then,

$$
S=\left\{x \in E: g_{j}(x) \leq 0, \quad j=1, \cdots, m\right\},
$$

is semi-connected with respect to $\eta(x, y, \theta)$.

Proof. Let $x, y \in S$, then we have $x, y \in E$ and $\max \left\{g_{j}(x), g_{j}(y)\right\} \leq 0$ for all $j \in\{1, \cdots, m\}$. Note that $g_{j}(x)$ are semi-prequasi-invex functions on $E$ with respect to the vector-valued function $\eta(x, y, \theta)$, then for any $\theta \in[0,1]$ and $j \in\{1, \cdots, m\}$, we get

$$
g_{j}(y+\theta \eta(x, y, \theta)) \leq \max \left\{g_{j}(x), g_{j}(y)\right\} \leq 0,
$$

which implies that $y+\theta \eta(x, y, \theta) \in S$. Therefore, $S$ is semi-connected with respect to $\eta(x, y, \theta)$. This completes the proof.

\section{Alternative Theorems and Optimality Condition For Multiobjective Optimization Problem}

In this section, we first establish two alternative theorems, then, we discuss an optimality necessary condition for multiobjective optimization problem with inequality constraints by using the alternative theorems.

Consider the following inequality constrained multiobjective optimization problem $(C M P)$.

$$
\begin{aligned}
(C M P): & \min f(x)=\left(f_{1}(x), \cdots, f_{p}(x)\right)^{T} \\
& \text { s.t. } g(x) \leq 0, x \in E,
\end{aligned}
$$

where $f_{i}: E \rightarrow R(i=1, \cdots, p), g_{j}: E \rightarrow R(j=1, \cdots, m)$, are real-valued functions, $E \subseteq R^{n}$ is a semi-connected set with respect to $\eta: E \times E \times[0,1] \rightarrow E$ and $S=\left\{x \in E: g_{j}(x) \leq 0, i=1, \cdots, m\right\}$ denotes the feasible set of $(C M P)$.

Throughout this section, let

$$
\begin{aligned}
& R_{+}^{m}=\left\{x \in R^{m} \mid x=\left(x_{1}, \cdots, x_{m}\right), x_{i} \geq 0,1 \leq i \leq m\right\}, \\
& R_{++}^{m}=\left\{x \in R^{m} \mid x=\left(x_{1}, \cdots, x_{m}\right), x_{i}>0,1 \leq i \leq m\right\} .
\end{aligned}
$$

Then, we recall the definitions of efficient solution and weakly efficient solution.

Definition 6. (see [9]) A point $x \in S$ is called a global efficient solution of $(C M P)$, if there does not exist any point $y \in S$, such that

$$
f(y) \in f(\bar{x})-R_{+}^{m} \backslash\{0\} .
$$

A point $\bar{x} \in S$ is called a local efficient solution of $(C M P)$, if there is a neighborhood $N(\bar{x})$ of $\bar{x}$, such that there does not exist any point $y \in S \cap N(\bar{x})$, such that

$$
f(y) \in f(\bar{x})-R_{+}^{m} \backslash\{0\} .
$$


Definition 7. (see [9]) A point $\bar{x} \in S$ is called a global weakly efficient solution of $(C M P)$, if there does not exist any point $y \in S$, such that

$$
f(y) \in f(\bar{x})-R_{++}^{m} .
$$

A point $\bar{x} \in S$ is called a local weakly efficient solution of $(C M P)$, if there is a neighborhood $N(\bar{x})$ of $\bar{x}$, such that there does not exist any point $y \in S \cap N(\bar{x})$, s.t.

$$
f(y) \in f(\bar{x})-R_{++}^{m} .
$$

Similarly to Lemma 1 in [3], we obtain the Lemma 8 as follows, which will be used later.

Lemma 8. Let $E$ be a semi-connected set of $R^{n}$, and $f_{i}(x), i=1, \cdots, m$, be semi-prequasi-invex functions. Then exactly one of the following two systems is solvable:

(i) there exists $\bar{x} \in E$, s.t. $f_{1}(\bar{x})<0, \cdots, f_{m}(\bar{x})<0$;

(ii) there exists $\lambda \in R_{+}^{m} \backslash\{0\}$, s.t. $\sum_{i=1}^{m} \lambda_{i} f_{i}(x) \geq 0, \forall x \in E$.

In the sequel, by using the above lemma we give the following Lemma 9 .

Lemma 9. Let $E \subseteq R^{n}$ be a nonempty compact semi-connected set with respect to $\eta: E \times E \times[0,1] \rightarrow E$, and $f_{i}(x), i \in M$, be lower semi-continuous semi-prequasi-invex functions with respect to the same $\eta(x, y, \theta)$ on $E$, where $M$ is finite or infinite index sets. Then, exactly one of the following two systems holds:

(i) $f_{i}(x) \leq 0, i \in M$ have a solution on $E$;

(ii) there exists $m \geq 1,\left\{i_{1}, \cdots, i_{m}\right\} \subseteq M$ and $p \in R_{+}^{m} \backslash\{0\}$, s.t.

$$
\sum_{j=1}^{m} p_{i} f_{i_{j}}(x)>0, \text { for all } x \in E .
$$

Proof. Obviously, (ii) does not hold when (i) holds. We only need to show that (ii) holds when (i) does not hold.

Now, we claim that there exists sufficient small $\bar{\varepsilon}>0$, such that $f_{i}(x) \leq \bar{\varepsilon}, i \in M$, have no solution on $E$. In the sequel, we testify the above claim.

By contradiction, suppose that there exists a positive real number sequence $\left\{\varepsilon_{k}\right\}, k=1,2, \cdots$, with $\lim _{k \rightarrow \infty} \varepsilon_{k}=0$, and for any $\varepsilon_{k}>0$, there exists $x_{k} \in E$, such that

$$
f_{i}\left(x_{k}\right) \leq \varepsilon_{k}, \forall i \in M
$$

Note that $E$ is a compact set, then the sequence $\left\{x_{k}\right\}$ must include a convergence subsequence, without loss of generality, we may assume that $\left\{x_{k}\right\}$ is the convergence subsequence and converges to $\bar{x}$. And $\bar{x} \in E$ due to the closeness of $E$. It follows from the lower semi-continuity of $f_{i}(x)$ that

$$
f_{i}(\bar{x}) \leq 0, \forall i \in M
$$

which is a contradiction. This verifies the above claim.

Then, for any $\varepsilon>0$, let

$$
X(i, \varepsilon)=\left\{x \in E \mid f_{i}(x) \leq \varepsilon\right\} .
$$

It follows from the above claim that there exists $\bar{\varepsilon}>0$, such that

$$
\bigcap_{i \in M} X(i, \bar{\varepsilon})=\emptyset
$$

The lower semi-continuity of $f_{i}(x)(\forall i \in M)$ on $E$ and the closeness of $E$ implies that $X(i, \bar{\varepsilon})$ is closed. Moreover, by the finite intersection theorem, there must exist $m \geq 1,\left\{i_{1}, \cdots, i_{m}\right\} \subseteq M$, such that

$$
\bigcap_{j=1}^{m} X\left(i_{j}, \bar{\varepsilon}\right)=\emptyset
$$


which implies that the following systems have no solution,

$$
f_{i_{j}}(x) \leq \bar{\varepsilon}, j=1, \cdots, m, \text { for all } x \in E \text {. }
$$

Clearly, the following systems also have no solution,

$$
f_{i_{j}}(x)<\bar{\varepsilon}, j=1, \cdots, m, \text { for all } x \in E,
$$

Note that $f_{i_{j}}(x), \forall j=1,2, \cdots, m$ is semi-prequasi-invex function. This fact together with Lemma 8 yields that there must exist $p \in R_{+}^{m} \backslash\{0\}$, such that

$$
\sum_{j=1}^{m} p_{i_{j}}\left(f_{i_{j}}(x)-\bar{\varepsilon}\right) \geq 0, \text { for all } x \in E
$$

and so,

$$
\sum_{j=1}^{m} p_{i_{j}} f_{i_{j}}(x) \geq \sum_{j=1}^{m} p_{i_{j}} \bar{\varepsilon}>0, \text { for all } x \in E \text {. }
$$

The proof is complete.

Remark 10. If $M$ is finite index sets in the above lemma, without loss of generality, we suppose that $M=\{1,2, \cdots, n\}$, then the lemma holds for $m=n$.

Next, we define a numerical valued optimization problem $(C M P)_{\lambda}$ as follows, which is relevant to $(C M P)$.

$$
\begin{array}{ll}
(C M P)_{\lambda}: & \min \lambda^{T} f(x) \\
& \text { s.t. } g(x) \leq 0, x \in E,
\end{array}
$$

where $\lambda \in R^{p}, f(x), g(x), E$ are the same with $(C M P)$.

Theorem 11. Let $E \subseteq R^{n}$ be a semi-connected set with respect to $\eta: E \times E \times[0,1] \rightarrow E$, assume $f_{i}(x): E \rightarrow R(i=1, \cdots, p), g_{j}(x): E \rightarrow R(j=1, \cdots, m)$, are semi-prequasi-invex functions on $E$ with respect to the same $\eta(x, y, \theta)$. If $\bar{x}$ is a weakly efficient solution (efficient solution) of $(C M P)$. Then, there exists a vector $\lambda \in R_{+}^{p} \backslash\{0\}$, such that $\bar{x}$ is an optimal solution of $(C M P)_{\lambda}$.

Proof. Let $S=\left\{x \in E: g_{i}(x) \leq 0, i=1,2, \cdots, m\right\}$, by the Theorem 5 and $g_{i}(x): E \rightarrow R^{n}(i=1, \cdots, m)$, being semi-prequasi-invex functions on $E$ with respect to the same $\eta(x, y, \theta)$, one can get that $S$ is a semi-connected set with respect to $\eta(x, y, \theta)$. Since $\bar{x}$ is a weakly efficient solution of $(C M P)$, it follows that the following systems do not hold on $S$.

$$
f_{j}(x)-f_{j}(\bar{x})<0, j=1,2, \cdots, p .
$$

It follows from Lemma 8, together with the semi-prequasi-invexity of $f_{i}(x): E \rightarrow R$ on $E$ with respect to the same $\eta(x, y, \theta)$, that there exists $\lambda \in R_{+}^{p} \backslash\{0\}$, such that

$$
\sum_{j=1}^{p} \lambda_{j}\left(f_{j}(x)-f_{j}(\bar{x})\right) \geq 0, \forall x \in S
$$

or equivalently,

$$
\sum_{j=1}^{p} \lambda_{j} f_{j}(x) \geq \sum_{j=1}^{p} \lambda_{j} f_{j}(\bar{x}), \forall x \in S
$$

This completes the proof. 


\section{Strong Duality Theorem and Saddle Point Theorem For Generalized Nonlinear Fractional Programming}

In this section, we first recall some definitions and lemmas for semi-prequasi-invex type generalized nonlinear fractional programming ( in short, $(G N F P)$ ). Then, we show the strong duality theorem and saddle point theorem for $(G N F P)$, which improve and generalize the corresponding results in [13] and [16].

Throughout this section, we assume that $\|\cdot\|$ denotes $l_{1}$-norm and

$$
\begin{aligned}
& F(x)=\left(f_{1}(x), \cdots, f_{p}(x)\right)^{T}, \\
& G(x)=\left(g_{1}(x), \cdots, g_{p}(x)\right)^{T}, \\
& H(x)=\left(h_{1}(x), \cdots, h_{p}(x)\right)^{T} .
\end{aligned}
$$

Now, we consider the generalized nonlinear fractional programming problem as follows:

$$
(G N F P): \quad \bar{\theta}=\inf _{x \in S} \max _{1 \leq i \leq p}\left\{\frac{f_{i}(x)}{g_{i}(x)}\right\},
$$

where $f_{i}(x): E \rightarrow R, g_{i}(x): E \rightarrow R, \forall x \in E, g_{i}(x)>0(i=1, \cdots, p), h_{j}(x): E \rightarrow R(j=$ $1, \cdots, m), E \subseteq R^{n}, S=\left\{x \in E: h_{j}(x) \leq 0, j=1, \cdots, m\right\} \neq \emptyset$. Clearly, the feasible set $S \neq \emptyset$, which implies that $\bar{\theta}<+\infty$.

To obtain the dual theory for $(G N F P)$, let us recall Definition 12 and Lemma 13 as follows(for more details, see[13] and [16]).

Definition 12. For $x \in E, \mu \in R_{+}^{p},\|\mu\|=1$ and $v \in R_{+}^{m}$, we denote

$$
\begin{gathered}
G L(x, \mu, v)=\frac{\mu^{T} F(x)+v^{T} H(x)}{\mu^{T} G(x)}, \\
G E(x, v)=\max _{1 \leq i \leq p} \frac{f_{i}(x)}{g_{i}(x)}+\sum_{j=1}^{m} v_{j} \max _{1 \leq i \leq p} \frac{h_{j}(x)}{g_{i}(x)} .
\end{gathered}
$$

Let

$$
\phi_{1}(\mu, v)=\inf _{x \in E} G L(x, \mu, v), \phi_{2}(v)=\inf _{x \in E} G E(x, v),
$$

then, we define two duals of the problem (GNFP):

$$
\begin{aligned}
& (G N F D)_{1}: \sup _{\mu \in R_{+}^{m} \backslash\{0\}, v \in R_{+}^{m}} \phi_{1}(\mu, v), \\
& (G N F D)_{2}: \sup _{v \in R_{+}^{m}} \phi_{2}(v) .
\end{aligned}
$$

Next lemma(for more details, [13] and [16]) gives the weak duality relationship between $(G N F D)_{1}$ and $(G N F P),(G N F D)_{2}$ and $(G N F P)$.

\section{Lemma 13.}

(i) If $x \in S$, then for any $\mu \in R_{+}^{p},\|\mu\|=1$ and $v \in R_{+}^{m}$, we obtain

$$
\phi_{1}(\mu, v) \leq \max _{1 \leq i \leq p} \frac{f_{i}(x)}{g_{i}(x)}, \phi_{2}(v) \leq \max _{1 \leq i \leq p} \frac{f_{i}(x)}{g_{i}(x)} .
$$

(ii) Suppose that $v\left((G N F D)_{i}\right), i \in\{1,2\}$, is the optimal value of $(G N F D)_{i}, i \in\{1,2\}$, then we can get $v\left((G N F D)_{2}\right)=\bar{\theta}$ if $v\left((G N F D)_{1}\right)=\bar{\theta}$.

(iii) Suppose that $\bar{x}$ is an optimal solution of $(G N F P)$, then $\bar{x}$ is a weakly efficient solution of the system $(T G N F P)_{1}$, where

$$
\begin{aligned}
(T G N F P)_{1}: \quad & \min (F(x)-\bar{\theta} G(x)) \\
& H(x) \leq 0, x \in E .
\end{aligned}
$$


Remark 14. One can see that if $\bar{\theta}=-\infty$, then $v\left((G N F D)_{1}\right)=v\left((G N F D)_{2}\right)=-\infty$. Therefore, we only need to consider the case that $+\infty>\bar{\theta}>-\infty$.

Theorem 15. Let $E \subseteq R^{n}$ be a nonempty compact semi-connected set with respect to $\eta: E \times E \times[0,1] \rightarrow E$. Assume $f_{i}(x)-\bar{\theta} g_{i}(x)(i=1, \cdots, p), h_{j}(x)(j=1, \cdots, m)$, are lower semi-continuous and semi-prequasiinvex functions on $E$ with respect to the same $\eta(x, y, \theta)$. Then, we have $v\left((G N F D)_{1}\right)=v\left((G N F D)_{2}\right)=\bar{\theta}$, and there exists $(\bar{\mu}, \bar{v})$, which is an optimal solution of $(G N F D)_{1}$.

Proof. Note that feasible set of $(G N F P)$ is nonempty, that is $S=\{x \in E: H(x) \leq 0\} \neq \emptyset$. It follows from $\bar{\theta}=\inf _{x \in S} \max _{1 \leq i \leq p}\left\{\frac{f_{i}(x)}{g_{i}(x)}\right\}$ that the following systems have no solution.

$$
\begin{aligned}
& f_{i}(x)-\bar{\theta} g_{i}(x)<0, i=1, \cdots, p \\
& h_{j}(x) \leq 0, j=1, \cdots, m, x \in E .
\end{aligned}
$$

which implies that for any $\varepsilon>0$, the following systems have no solution too.

$$
\begin{aligned}
& f_{i}(x)-\bar{\theta} g_{i}(x)+\varepsilon \leq 0, i=1, \cdots, p \\
& h_{j}(x) \leq 0, j=1, \cdots, m, x \in E .
\end{aligned}
$$

Note that $f_{i}(x)-\bar{\theta} g_{i}(x)(i=1, \cdots, p), h_{j}(x)(j=1, \cdots, m)$, are lower semi-continuous semi-prequasiinvex functions on $E$, which together with Remark 10, yield that there exists $\bar{\mu} \in R_{+}^{p}, \bar{v} \in R_{+}^{m},(\bar{\mu}, \bar{v}) \neq 0$, such that

$$
\bar{\mu}^{T}(F(x)-\bar{\theta} G(x)+\Theta)+\bar{v}^{T} H(x)>0, \text { for all } x \in E,
$$

where $\Theta=(\varepsilon, \cdots, \varepsilon) \in R_{++}^{p}$. Clearly, $S \neq \emptyset$ implies $\bar{\mu} \neq 0$. Without loss of generality, we may assume $\|\bar{\mu}\|=1$.

By $\forall x \in E, \bar{\mu}^{T} G(x)>0$ and (1), we have

$$
\frac{\bar{\mu}^{T} F(x)+\bar{v}^{T} H(x)}{\bar{\mu}^{T} G(x)}>\bar{\theta}-\frac{\varepsilon}{\bar{\mu}^{T} G(x)} .
$$

On the other hand,

$$
\frac{1}{\mu^{T} G(x)} \leq \frac{1}{\min _{1 \leq i \leq p} g_{i}(x)} \leq \frac{1}{\inf _{x \in E} \min _{1 \leq i \leq p} g_{i}(x)}=: \bar{\alpha}
$$

Then, (2) and (3) imply that $G L(x, \bar{\mu}, \bar{v})>\bar{\theta}-\varepsilon \bar{\alpha}$, for all $x \in E$. By the arbitrariness of $\varepsilon$, we have

$$
G L(x, \bar{\mu}, \bar{v}) \geq \bar{\theta}, \text { for all } x \in E .
$$

(4), together with Lemma 13 (i) and (ii), yields $v\left((G N F D)_{1}\right)=v\left((G N F D)_{2}\right)=\bar{\theta}$, and $\phi_{1}(\bar{\mu}, \bar{v})=$ $\inf _{x \in E} G L(x, \bar{\mu}, \bar{v})=\bar{\theta}$. Thus, the theorem is proved.

Next, let us give the saddle point theory for $G L(x, \mu, v)$.

Theorem 16. Let $E \subseteq R^{n}$ be a nonempty semi-connected set with respect to $\eta: E \times E \times[0,1] \rightarrow E$. Suppose $f_{i}(x)-\bar{\theta} g_{i}(x)(i=1, \cdots, p), h_{j}(x)(j=1, \cdots, m)$, are semi-prequasi-invex functions on $E$ with respect to the same $\eta(x, y, \theta)$. If $\bar{x}$ is an optimal solution of $(G N F P)$, and there exists $x^{\prime} \in E$, s.t. $H\left(x^{\prime}\right)<0$. Then, there exist $\bar{\mu} \in R_{+}^{p} \backslash\{0\}, \bar{v} \in R_{+}^{m}$, such that $(\bar{x}, \bar{\mu}, \bar{v})$ is a saddle point of $G L(x, \mu, v)$ on $E \times R_{+}^{p} \backslash\{0\} \times R_{+}^{m}$, that is, for $\forall x \in E, \forall \mu \in R_{+}^{p} \backslash\{0\}, \forall v \in R_{+}^{m}$, we have

$$
G L(\bar{x}, \mu, v) \leq G L(\bar{x}, \bar{\mu}, \bar{v}) \leq G L(x, \bar{\mu}, \bar{v}),
$$

where

$$
G L(x, \mu, v)=\frac{\mu^{T} F(x)+v^{T} H(x)}{\mu^{T} G(x)} .
$$


Proof. $\bar{\theta}$ is an optimal value of $(G N F P)$, it implies that the following systems have no solution on $E$.

$$
\begin{aligned}
& f_{i}(x)-\bar{\theta} g_{i}(x)<0, \quad i \in\{1, \cdots, p\} \\
& h_{j}(x)<0, \quad j \in\{1, \cdots, m\} .
\end{aligned}
$$

$f_{i}(x)-\bar{\theta} g_{i}(x)(i=1, \cdots, p), h_{j}(x)(j=1, \cdots, m)$, are semi-prequasi-invex functions on $E$ with respect to the same $\eta(x, y, \theta)$, which together with Lemma 8, yield that there must exist $(\bar{\mu}, \bar{v}) \in R_{+}^{p} \times R_{+}^{m},(\bar{\mu}, \bar{v}) \neq$ 0 , such that

$$
\bar{\mu}^{T}(F(x)-\bar{\theta} G(x))+\bar{v}^{T} H(x) \geq 0, \text { for all } x \in E .
$$

Next, we prove that $\bar{\mu} \neq 0$. Otherwise, taking $x=x^{\prime}$ into (5), we have $\bar{v}^{T} H\left(x^{\prime}\right) \geq 0$, which contradicts with $x^{\prime} \in E$, s.t. $H\left(x^{\prime}\right)<0$. Obviously, we derive from $G(x)>0, \bar{\mu} \in R_{+}^{p} \backslash\{0\}$ that $\bar{\mu}^{T} G(x)>0$, which together with (5) lead to

$$
G L(x, \bar{\mu}, \bar{v})=\frac{\bar{\mu}^{T} F(x)+\bar{v}^{T} H(x)}{\bar{\mu}^{T} G(x)} \geq \bar{\theta} .
$$

Taking $x=\bar{x}$ into (5), it follows that

$$
\bar{\mu}^{T}(F(\bar{x})-\bar{\theta} G(\bar{x}))+\bar{v}^{T} H(\bar{x}) \geq 0 .
$$

However, $F(\bar{x})-\bar{\theta} G(\bar{x}) \leq 0, \bar{\mu} \in R_{+}^{p}, \bar{v} \in R_{+}^{m}$ imply that

$$
\bar{\mu}^{T}(F(\bar{x})-\bar{\theta} G(\bar{x}))+\bar{v}^{T} H(\bar{x}) \leq 0 .
$$

(7) and (8) imply that

$$
\bar{\mu}^{T}(F(\bar{x})-\bar{\theta} G(\bar{x}))+\bar{v}^{T} H(\bar{x})=0 .
$$

Clearly, $\bar{\mu}^{T} G(x)>0$, dividing (9) by $\bar{\mu}^{T} G(x)$, we obtain

$$
G L(\bar{x}, \bar{\mu}, \bar{v})=\frac{\bar{\mu}^{T} F(\bar{x})+\bar{v}^{T} H(\bar{x})}{\bar{\mu}^{T} G(\bar{x})}=\bar{\theta} .
$$

On the other hand, for $\forall \mu \in R_{+}^{p}, \forall v \in R_{+}^{m}$, we have

$$
\mu^{T}(F(\bar{x})-\bar{\theta} G(\bar{x}))+v^{T} H(\bar{x}) \leq 0 .
$$

Note that $\mu^{T} G(\bar{x})>0$, for all $\mu \in R_{+}^{p}$, then dividing (11) by $\mu^{T} G(\bar{x})$, we get

$$
G L(\bar{x}, \mu, v)=\frac{\mu^{T} F(\bar{x})+v^{T} H(\bar{x})}{\mu^{T} G(\bar{x})} \leq \bar{\theta} .
$$

It follows from (6), (10) and (12) that for $\forall x \in E, \forall \mu \in R_{+}^{p} \backslash\{0\}, \forall v \in R_{+}^{m}$, we have

$$
G L(\bar{x}, \mu, v) \leq G L(\bar{x}, \bar{\mu}, \bar{v}) \leq G L(x, \bar{\mu}, \bar{v}) .
$$

This completes the proof.

\section{Conclusions}

In this paper, we mainly study semi-prequasi-invex type multiobjective optimization problem with inequality constraints and generalized nonlinear fractional programming. We first discuss two alternative theorems and an optimality necessary condition for multiobjective optimization problem. Then, we establish a strong duality theorem and a saddle point theorem for generalized nonlinear fractional programming. Our results improve and generalize the corresponding ones in the literature.

An interesting topic for our future research is to investigate more weaker assumptions to study the applications of semi-prequasi-invexity in vector optimization. 
Acknowledgments. This research was partially supported by the National Natural Science Foundation of China (11301571,11401058), the Basic and Advanced Research Project of Chongqing(2015jcyjA00025,2016jcyjA0219), the China Postdoctoral Science Foundation funded project (2015M580774,2016T90837) and Chongqing Graduate Research and Innovative Training Program (Grant No. CYS16144).

\section{References}

1. A. Ben-Israel and B. Mond, "What is invexity?" The Journal of the Australian Mathematical Society, Series $B$, vol. 28 , no. 1, pp. 1-9, 1986.

2. D. H. Martin, "The essence of invexity," Journal of Optimization Theory and Applications, vol. 47, no. 1, pp. 65-76, 1985.

3. X. Q. Yang and G. Y. Chen, "A class of nonconvex functions and pre-variational inequalities," Journal of Mathematical Analysis and Applications, vol. 169, no. 2, pp. 359-373, 1992.

4. X. M. Yang and D. Li, "On properties of preinvex functions," Journal of Mathematical Analysis and Applications, vol. 256, no. 1, pp. 229-241, 2001.

5. —_ "Semistrictly preinvex functions," Journal of Mathematical Analysis and Applications, vol. 258, no. 1, pp. 287-308, 2001.

6. Z. Y. Peng, K. K. Li, and S. S. Zhang, "D- $\eta$-E-semipreinvex vector mappings and vector optimization," Applied Mathematics and Mechanics, vol. 35, no. 9, pp. 1020-1032, 2014(In Chinese).

7. K. K. Li, Z. Y. Peng, X. Wan, and P. Tang, "The study of strict G-semi-preinvexity and its applications semistrictly preinvex functions," Journal of Chongqing Normal University (Edition of Natural Science), vol. 32, no. 6, pp. 1-8, 2015(In Chinese).

8. X. M. Yang, "A note on preinvexity," Journal of Industrial and Management Optimization, vol. 10, no. 4, pp. 1319-1321, 2014.

9. — "Problems of semi-preinvexity and multi-objective programming," Journal of Chongqing Normal University (Edition of Natural Science), vol. 11, no. 1, pp. 1-5, 1994(In Chinese).

10. X. M. Yang, X. Q. Yang, and K. L. Teo, "Characterizations and applications of prequasi-invex functions," Journal of Optimization Theory and Applications, vol. 110, no. 3, pp. 645-668, 2001.

11. H. Z. Luo and Z. K. Xu, "Technical note on characterization of prequasi-invex functions," Journal of Optimization Theory and Applications, vol. 120, no. 2, pp. 429-439, 2004.

12. Z. Y. Peng, K. K. Li, P. Tang, and Y. Q. Huang, "Characterizations and criterions of d-semiprequasi-invex mappings," Journal of Chongqing Normal University (Edition of Natural Science), vol. 31, no. 5, pp. 18-25, 2014(In Chinese).

13. Y. X. Zhao, "A type of generalized convexity and applications in optimization theory," Master Degree Thesis. Jinhua: Zhejiang Normal University, 2005(In Chinese).

14. Y. X. Zhao, X. G. Meng, H. Qiao, S. Y. Wang, and L. Coladasuria, "Characterizations of semi-prequasi-invexity," Journal of Systems Science and Complexity, vol. 27, no. 5, pp. 1008-1026, 2014.

15. S. K. Mishra, "Topics in nonconvex optimization: Theory and applications," Springer Optimization and Its Applications, Springer, New York, USA, vol. 50, 2011.

16. Z. K. Xu, "Duality in generalized nonlinear fractional programming," Journal of Mathematical Analysis and Applications, vol. 169, no. 1, pp. 1-9, 1992. 